\title{
Surface Acoustic Wave Induced Heat Knockdown of Caenorhabditis Elegans
}

\author{
Adem Ozcelik (iD \\ Aydin Adnan Menderes University, Department of Mechanical Engineering, Aydın, Turkey
}

\section{A BSTRACT}

$\mathrm{M}$ odel organisms such as Caenorhabditis elegans are commonly used in discovering the underlying mechanisms of human diseases. The field of microfluidics enables a plethora of tools for better studying these organisms. In this research, an effective and simple approach is demonstrated for thermally induced knockdown of Caenorhabditis elegans model organisms. To achieve this, surface acoustic waves are coupled to the fluid medium inside a microfluidic channel. Without active cooling, two pairs of propagating surface acoustic waves induce very rapid heating of the enclose fluid. By controlling the input power of the acoustic waves, temperature of the fluid is precisely adjusted, and induced reversible knockdown of Caenorhabditis elegans samples. Effective temperature window of acoustically induced thermal knockdown is characterized, and the tolerable number of knockdown and wake up cycles is studied. Viability of the Caenorhabditis elegans organisms is also evaluated after increasing numbers of knockdown cycles. Overall, a simple and effective method of worm immobilization method is demonstrated using acoustic waves in microfluidics.

\section{Keywords:}

C. elegans, Immobilization, Microfluidics, Surface acoustic waves, Thermoacoustics

\author{
Article History: \\ Received: 2020/10/04 \\ Accepted: 2021/02/04 \\ Online: 2021/03/31 \\ Correspondence to: Adem Özçelik \\ Department of Mechanical Engineering, \\ Aydin Adnan Menderes University, \\ Aydın, Turkey \\ Tel: +905350465266 \\ Fax: +902562136686 \\ E-Mail:aozcelik@adu.edu.tr
}

\section{INTRODUCTION}

$\mathrm{C}$ aenorhabditis elegans (C. elegans) is a versatile model organism used for studying various human diseases due to its easy maintenance, adequate complexity in terms of biological functionality, and short life cycle allowing observation of its entire developmental cycle in a week [1,2]. Because of its small size, which is around $1 \mathrm{~mm}$ in adulthood, microfluidic platforms have been widely adopted for studying C. elegans [3,4]. Microfluidics is a relatively new field which is targeting miniaturization of bioanalysis, chemical synthesis, sample processing, and disease diagnostics [5-9]. For C. elegans related research, microfluidics provides small and well-controlled micro environments enabling convenient microscopic investigation [10,11]. However, in order to precisely image cellular and subcellular structures of these organisms, they are required to be immobilized during optical imaging.

There are three general approaches for steadily observing and imaging the cellular details of this organism which are chemically, physically and thermally induced immobilization $[12,13]$. In chemical immobilization, various chemicals including levamisole and sodium azide are used to sedate the worms similar to the anesthetics administer in surgical procedures [14]. Even though, chemical immobilization works quickly and effectively, their biological effects on the worm metabolism is not clear [15]. For biological research in which no foreign biochemical interference is desired, administering these chemicals is not suitable. The second approach is physical immobilization through restricting the motion of the worms by using various microfluidic methods [3]. One of these methods is using thin membrane based suction to physically immobilize a worm inside a microfluidic channel [16]. These methods generally require complex microfluidic device fabrication which is not available in many laboratories in low-budget institutions. Microfluidic immobilization also imposes a predefined geometry which can obstruct clear manipulation and/or observation of the worms. Finally, thermal worm immobilization can be applied either by heating or cooling the worms to specific temperatures to minimize their metabolic activity or induce thermal shock, respectively [17]. In cooling approach, worms take a curled shape minimizing their body length which renders detailed microscopic imaging more challenging. For the temperature induced heat shock worms are immobilized in a temperature controlled environment. In one example, a laser is applied to induce rapid temperature increase inside a multi-layered fluidic device [18]. This 
method proves effective worm immobilization, but device fabrication and operation are complicated due to the required multi-layer conductive channels and application of lasers. In addition, application of a single laser spot makes this method low throughput. Considering the challenges and disadvantages of the existing worm immobilization methods, there is still a need for a simpler and more practical method that can provide effective and high throughput worm immobilization.

In this work, a standing surface acoustic wave (SSAW)based microfluidic method is demonstrated for inducing controllable heat knockdown of C. elegans worms. Acoustic methods enable a versatile platform in many fields $[19,20]$. The method presented in this work relies on acoustically generated rapid temperature increase inside a microfluidic channel. C. elegans worms are repeatedly induced into recoverable heat shocks, and their endurances and recovering periods are characterized. Overall, as a potential method for worm researchers, a SSAW-based method is applied in C. elegans immobilization for the first time in literature.

\section{MATERIAL AND METHODS}

Device fabrication is completed in two steps. In the first step, interdigitated transducers (IDTs) are patterned on a lithium niobate $\left(\mathrm{LiNbO}_{3}\right)$ piezoelectric wafer which is a transparent, $128^{\circ}$ Y-cut piezoelectric crystal. Standard photolithography is applied to fabricate the gold electrodes of the IDTs. For this, initially a positive photoresist (Megaposit ${ }^{\mathrm{ma}} \mathrm{SPR}^{\mathrm{rm}} 220$ ) is spin-coated on the $\mathrm{LiNbO}_{3}$ wafer. Then, a photomask with the desired electrode pattern is used to expose the photoresist, and subsequently developed in the resist developer. Afterwards, $5 \mathrm{~nm}$ of chromium and $200 \mathrm{~nm}$ of gold layers are coated on top of the patterned wafer via vacuum thermal evaporation. Finally, coated wafer is incubated in acetone for overnight to liftoff the gold layers coated on the remaining photoresist regions. General steps of IDT fabrication are shown in Fig 1. The design of the IDTs includes $50 \mu \mathrm{m}$ wide electrodes separated by $50 \mu \mathrm{m}$ distance. The design of the IDTs is chosen to be straight to uniformly cover the width of the microfluidic channel. The wavelength of the SAWs corresponds to four times the width of the electrodes of IDTs. Using the speed of sound in $128^{\circ}$ Y-cut LiNbO3 at room temperature $(3980 \mathrm{~m} / \mathrm{s})$ and the wave frequency conversion equation $(\mathrm{f}=\mathrm{v} / \lambda$, where $\mathrm{f}$ is the frequency, $\mathrm{v}$ is the speed of sound and $\lambda$ is the wavelength), we can find the resonance frequency of the fabricated IDTs for $50 \mu \mathrm{m}$ electrodes to be around $20 \mathrm{MHz}$.

In the second step, a microfluidic channel is fabricated via soft lithography process. Soft lithography refers to a precise replica molding method. For this step, a master mold containing the positive features of the microfluidic channel is fabricated through photolithography and deep reactive ion etching (DRIE) processes [21]. Briefly, a silicon wafer is spin coated with the positive photoresist, and exposed with a photomask. Then developed and bake at $90{ }^{\circ} \mathrm{C}$ for about 20 minutes. The silicon wafer now contains photoresist coated regions where microfluidic channel features are located. The rest of the silicon wafer surface is just bare silicon. DRIE process is applied to this wafer to etch the non-resist coated regions to a depth of 100 micrometers which defines the microfluidic channel height. 100-micrometer channel height is chosen to allow unrestricted infusion of larger worms into the fluidic channel preventing potential device clogging. DRIE etched wafer is then washed with acetone to clear to rest of the resist covering the un-etched regions.

Once the silicon master mold is fabricated, a mixture of Polydimethylsiloxane (PDMS) is poured on top of the mold surface. After curing at $60{ }^{\circ} \mathrm{C}$ for around 2 hours, the liquid PDMS solidifies and forms a transparent elastomer. The microfluidic channels are peeled and cut to smaller devices. Then, inlets and outlets of the PDMS device is punched to enable polyethylene tube and thermocouple insertion. The port for the thermocouple probe is made slightly smaller than the actual thermocouple tip to avoid any potential leakage. Finally, the PDMS device is bonded to LiNbO3 using plasma activation of both surfaces, and overnight baking at $60^{\circ} \mathrm{C}$.

The SSAW-based microfluidic device is driven by an arbitrary signal generator (AFG1062, Tektronix). The imaging of the worms is performed using an inverted microscope equipped with a high definition camera (OX.2053-PLPH, Euromex). Wild type $\mathrm{C}$. elegans worms are grown in agar medium at $20^{\circ} \mathrm{C}$, and Escherichia coli (OP50) is used as the nutrition source [11]. Worms are collected in an M9 buffer solution for conducting the microfluidic experiments. Worms are delivered into the microfluidic device through a custom designed automated syringe pump. Temperature inside the channel is measured by a fine tip temperature probe (TJ Probe, Omega).

To evaluate the viability of the worms after each knockdown cycle, the worms within the active acoustic treatment region are collected in a fresh M9 solution. To avoid collecting non-treated worms, inlet and outlet tubing of the device are replaced with the new tubes in each cycle. Then, $20 \mu \mathrm{M}$ propidium iodide solution is added to $200 \mu \mathrm{L}$ worm solution. After the staining, the worms are collected in a petri dish containing agar plate. The collected worms are observed for motility, and propidium iodide stain. The scoring of the live and dead worms is completed under the microscope. 


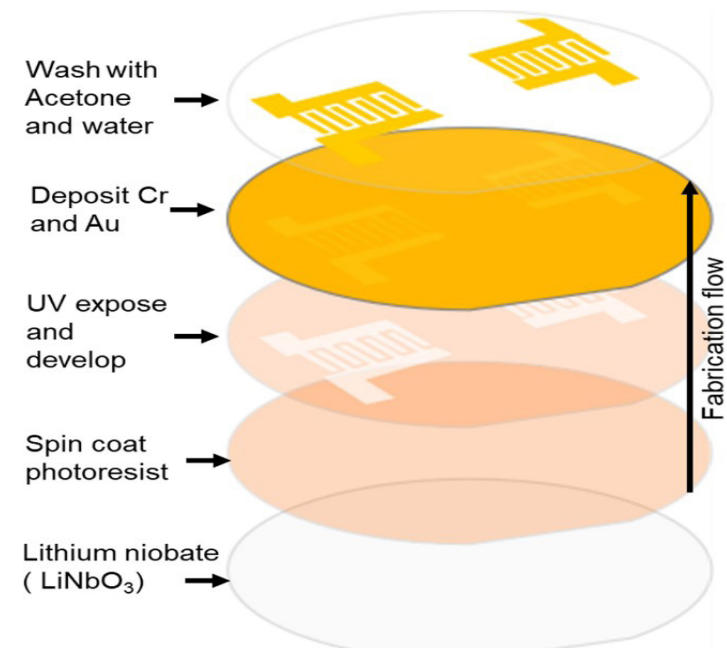

Figure 1. Fabrication of IDTs on a lithium niobate substrate. First, a positive photoresist is spin-coated on the lithium niobate wafer. Second the coated surface of the wafer is UV exposed through a photomask with the desired IDT patterns, and the wafer is developed. Then $5 \mathrm{~nm}$ chromium and $200 \mathrm{~nm}$ gold is deposited on the lithium niobate surface.

\section{RESULTS AND DISCUSSION}

The schematic model and the actual standing surface acoustic wave-based microfluidic device are shown in Fig. 2. The microfluidic channel is designed to have curved inner surfaces to prevent microbubble trapping inside the channel. The inlet in the center area of the microfluidic channel is used to insert the temperature probe into the device. The two symmetrically fabricated IDTs are used to generate two travelling surface acoustic waves in opposite directions, and form standing waves inside the fluidic channel. The aim of using the SSAWs in this device is to amplify and provide evenly distributed thermoacoustic effects of the acoustic waves within the fluid volume inside the microfluidic channel. The tip of temperature probe tightly fits in the center inlet providing liquid tight connection. It is also important to note that the tip of the temperature probe is not touching to the lithium niobate surface to prevent additional heating that may be transferred through solid surfaces.

The correlation between the applied voltage and the medium temperature inside the microfluidic device is observed to be linear, and is given in Fig. 3. Here, the measurements are taken after the inner temperature is stabilized at a given applied voltage value. The experiments are repeated for 6 times for each voltage value to obtain adequate statistical data. Average value of the temperature readings from different experiments are plotted along with the standard deviations. The data in Fig. 3 is collected using a $20 \mathrm{MHz}$ SSAW device. One can also use a higher or lower resonant frequency device to induce a gradual temperature increase inside a microfluidic channel. The only difference between these devices would be the required applied voltage to reach the same temperature.
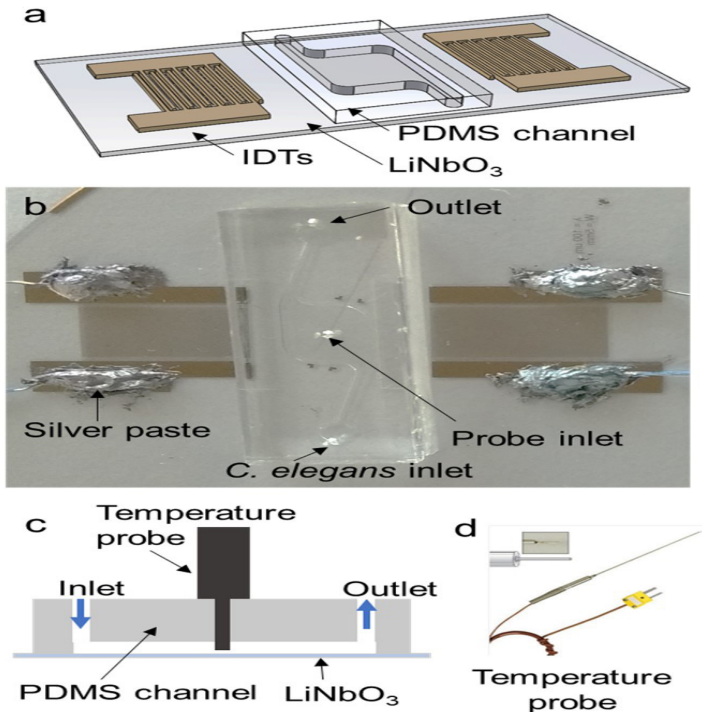

PDMS channel LiNbO

Figure 2. General overview of the device. a) Schematic and b) actual standing surface acoustic wave device. c) side view of the microfluidic device demonstrating the inlets and the outlets. d) picture of the fine tip temperature probe. The tip of the probe has a $0.5 \mathrm{~mm}$ diameter and 2.4 mm length.

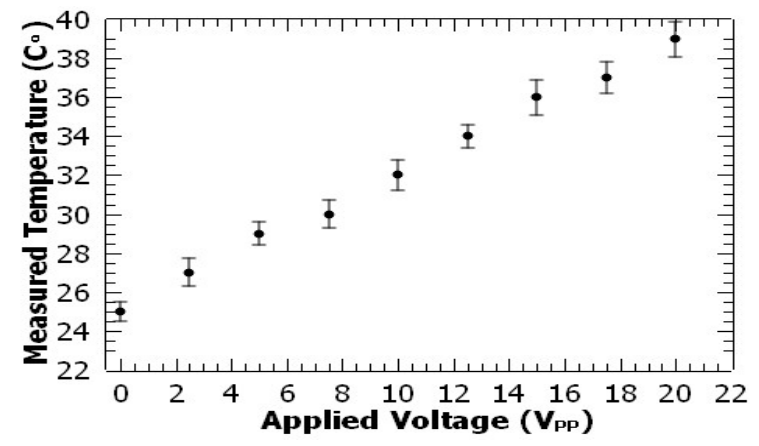

Figure 3. Experimental relationship between the applied voltage to the IDTs and the measured temperature inside the microfluidic channel. Experiments are started at room temperature. A linear relationship is observed between the applied voltage and the temperature. The error bars represent the standard deviation of 6 different measurements at each voltage value.
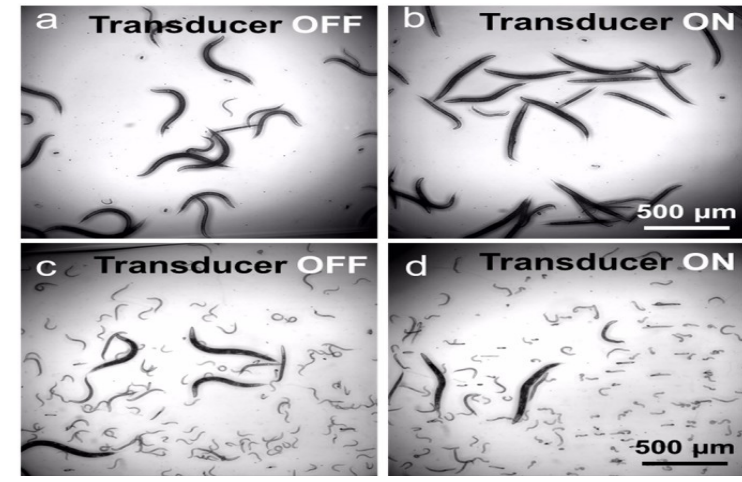

Figure 4. Experimental observation of $C$. elegans worm behavior under the effect of temperature rise in the presence of acoustic waves. a) Low and c) high concentrations of $C$. elegans are shown to be in their natural swimming body postures without any acoustic treatment. b) and d) When the acoustic waves are applied with 15 VPP, worms are immobilized with the effect of the heat shock, and their body posture becomes more elongated. 
An example C. elegans treatment experiment is demonstrated in Fig. 4. Both low concertation adult populations (Figs. 4a and b) and high concentration mixed development stage worms (Figs. c and d) are shown to be effectively immobilized by application of 15 VPP input voltage. In both cases, when the transducer is off, worms exhibit natural swimming body postures with curved shapes. In the M9 buffer, which is a liqud medium, C. elegans worms perform swimming motion via body undulations unlike crawling motion they do in more viscous and granular agar medium. Once the transducer is turned on, acoustic waves induce a rapid heating and subsequent temperature rise inside the fluidic chamber. C. elegans worms go through a thermal shock demonstrating seizure like body motions for few seconds and finally are knockdown. After heat induced knowckdown (Figs. 4b and d) body posture of the worms become more elongated similar to chemical immobilization. No observable difference is recorded in the thermal immobilization behavior of different developmental stage worms.

To better elucidate the effect acoustically generated heat on worm knockdown, the effective temperature window that worms goes through knockdown and become immobilized is studied. For these experiments, fresh batches of worm populations are introduced into the microfluidic chamber before each temperature setting. As seen in Fig. 3, the temperate of the fluid medium can be adjusted from 25 ${ }^{\circ} \mathrm{C}$ to $39^{\circ} \mathrm{C}$ by adjusting he applied voltage from 3 VpP to 20 VPP. Starting from the room temperature and going up to $39^{\circ} \mathrm{C}$, different worm batches are exposed to acoustically generated heat, and the state of the worms are studied from their physical movements. Results of these experiments are given in Fig. 5. As observed from the figure, below $32^{\circ} \mathrm{C}$, worms do not exhibit heat knockdown. Starting from $32^{\circ} \mathrm{C}$, worms start to go through the seizures and eventual knockdown. $35^{\circ} \mathrm{C}$ is found to be an effective temperature for rapid knockdown of worms. Above $37^{\circ} \mathrm{C}$, the effects of the temperature become detrimental to the worm biology, and worms die after the seizures. In Fig. 5, a knockdown window is demonstrated.

The C. elegans worms are heavily dependent on the environmental conditions including temperature fluctuations. Under the detrimental temperatures such as 38 ${ }^{\circ} \mathrm{C}$ and above, the neural circuitry of the worm turns off to protect the animal to a certain degree. The temperature window that is demonstrated in Fig. 5 outlines the range of temperatures where $C$. elegans can shut down some of its neural activity and receive no damage. Within this range of temperature, the worms can wake up once the excessive temperature is removed. The microfluidic environment enables very rapid heating of the worm medium, and similarly heat dissipation due to the small volumes of fluids. Acoustic waves can induce instantaneous temperature increase within the domain of the fluid media that the Rayleigh waves of the SAWs leak into.

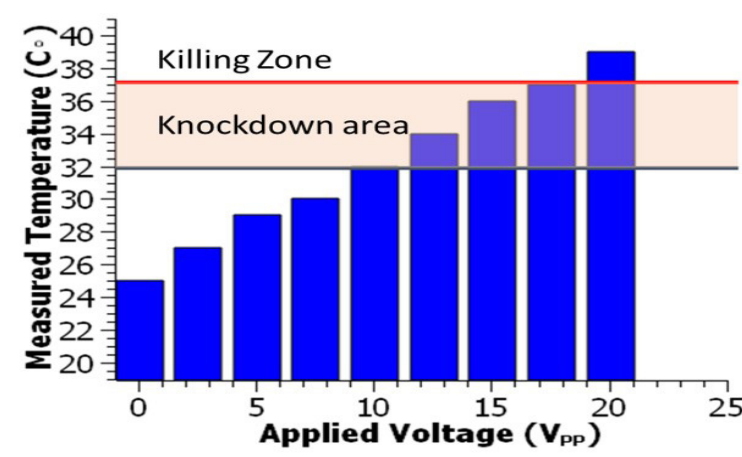

Figure 5. Demonstration of effective temperature window for worm immobilization. An interval between $32^{\circ} \mathrm{C}$ and $37^{\circ} \mathrm{C}$ is identified to induce heat knockdown of worms. Mixed population worm samples are used in these experiments.

In Fig. 6, the time required for knockdown and wake up of the C. elegans is demonstrated. Here, each experiment is conducted at $35{ }^{\circ} \mathrm{C}$. The quantification of the experiments is done by observation of complete knockdown and revival of the worm population exposed to the thermal treatment. For each worm sample in the microfluidic device, 10 cycles of rapid temperature increase and decrease are applied. In total 20 batches of worms are used, and each batch is cycled 10 times. Error bars are calculated from the standard deviation of the 20 different experiments for each cycle order. From this data set shown in Fig. 6, it is observed that within the first 4 cycles, the time required for the knockdown and wake up is fairly constant and less than 10 seconds for each. However, starting from the fifth cycle, wake up times are gradually increasing. This indicates that subsequent cycling of the same worms (continuous knockdown and wake up) adversely affect the worm physiology and require longer durations for wake up. Nevertheless, within the first five cycles, the required time for thermal immobilization and recovery is quite fast, and reasonable for the $6^{\text {th }}, 7^{\text {th }}$, and $8^{\text {th }}$ cycles which are under 100 seconds. Considering the chemical immobilization of $\mathrm{C}$. elegans which require minutes for the knockdown and wake up, the acoustically induced heat knockdown is more effective in terms of time requirements.

In worm experimentation using microfluidic channels, knockdown of worms several times can be a reasonable demand. According to Fig. 6, standing surface acoustic wave-based method can provide up to 8 immobilization cycling safely. In addition, when a continuous duration of immobilization is required for C. elegans operations and imaging, the knockdown induced temperature can be sustained for the entire duration of the study. After the operation is completed, induced elevated temperature can be removed by turning off the acoustic field. 


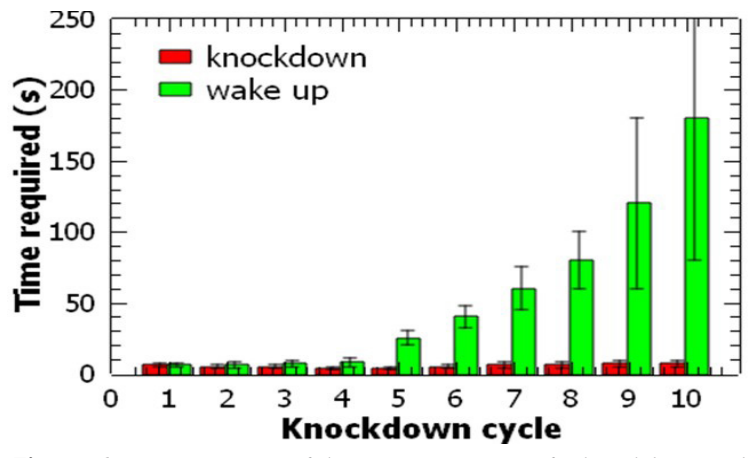

Figure 6. Demonstration of the time requirement for knockdown and wakeup after repeated cycles. After the first 4 cycles, it takes longer for the worm to recover from the effect of the knockdown. Each cycle is repeated 20 times for different batches of worms. Error bars represent the standard deviation of 20 data points for each cycle. Mixed population worm samples are used in these experiments.

After measuring the time required for the thermal immobilization of the worms, it is also important to study the detrimental effects of the knockdown and wake up cycles on worm biology. For this, the rate of viability is characterized after cycling the worms. Individual worms are counted to score the dead and live worms after each knockdown and wake up. In Fig. 7, the results of the scoring are given. According to the graph, worm viability is in the range of $95 \%$ within the first 4 cycles. In the 5 th and 6th cycles, the viability drops to around $85 \%$. After the 10th cycle, the worm viability becomes 50\%. Compare to the other worm immobilization methods including chemical and thermal, acoustically induced method enables both a high throughput and rapid immobilization method. For instance, using the method presented in this study (which can also be referred as the thermoacoustic method), worm immobilization can be applied anytime within he microfluidic channel whereas chemical treatment of the worms requires the administration of the chemical agents and certain time to allow sedation resulting in worm immobilization.

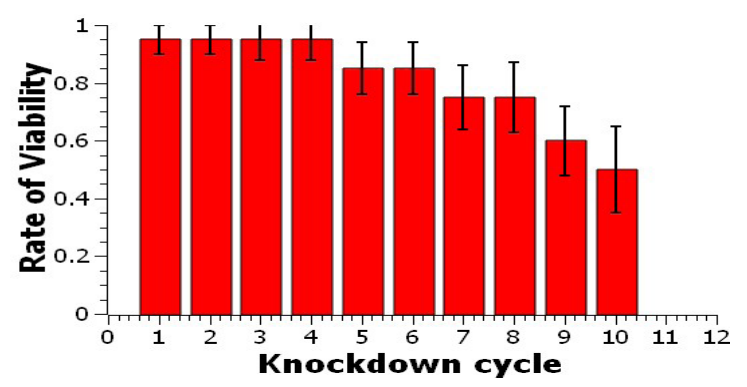

Figure 7. Demonstration of the effect of repeated knockdown cycles on worm viability. As a general trend, viability drops with the repeated number of cycles. Error bars are obtained from standard deviation of three different experiments for each cycle. Mixed population worm samples are used in these experiments.

Furthermore, with the acoustic method, the active region of the SSAW field, can be divided into smaller sections by fabricating smaller pairs of IDTs. This way, only a selected group of worms can be treated and immobilized. Thanks to the rapid heat dissipation occurring in the microfluidics, the induced temperature rise can be contained only within the active acoustic field.

\section{CONCLUSION}

Herein, a new method of C. elegans immobilization is demonstrated using a standing surface acoustic wavebased microfluidic device. Thermoacoustically induced rapid temperature rises are applied inside a microfluidic channel to temporarily immobilize worm samples. The time required for immobilization is found to be much smaller compared to the chemical methods. The acoustic method is also more advantages compared to the laser-driven thermal methods due to the high throughput and device simplicity enabled by the surface acoustic wave device. Overall, the method presented in this work can be a valuable tool for worm researchers due to its simplicity, versatility and adoptability into microfluidic platforms.

\section{ACKNOWLEDGEMENTS}

This research was supported by Adın Adnan Menderes University Research Fund. Project Number: MF-20002.

\section{REFERENCES}

1. Kaletta, T., Hengartner, M.O. Finding function in novel targets: C. elegans as a model organism. Nature Reviews. Drug Discovery. 5(5) (2006) 387-98. doi: 10.1038/nrd2031

2. Rohde, C.B., Zeng, F., Gonzalez-Rubio, R., Angel, M., Yanik, M.F. Microfluidic system for on-chip high-throughput whole-animal sorting and screening at subcellular resolution. Proceedings of the National Academy of Sciences of the United States of America. 104(35) (2007) 13891-5. doi: 10.1073/pnas.0706513104.

3. Gilleland, C.L., Rohde, C.B., Zeng, F., Yanik, M.F. Microfluidic immobilization of physiologically active Caenorhabditis elegans. Nature Protocols. 5(12) (2010) 1888-902. doi: 10.1038/ nprot.2010.143.

4. Cáceres, I. de C., Valmas, N., Hilliard, M.A., Lu, H. Laterally orienting $\mathrm{C}$. elegans using geometry at microscale for highthroughput visual screens in neurodegeneration and neuronal development studies. PLoS ONE. 7(4) (2012) e35037. doi: 10.1371/ journal.pone.0035037.

5. Tosun, E., Ozgur, T., Ozgur, C., Ozcanli, M., Serin, H., Aydin, $\mathrm{K}$. Comparative analysis of various modelling techniques for emission prediction of diesel engine fueled by diesel fuel with nanoparticle additives. European Mechanical Science. 1(1) (2017) 15-23. doi: 10.26701/ems.320490.

6. Akmeşe, B., Asan, A. Optimization of the Schiff-Base Reaction of Acetylacetone with Biogenic Amines. Hittite Journal of Science and Engineering. 4(1) (2017) 79-83. doi: 10.17350/ HJSE19030000052.

7. Erdoğan, B., Topuz, A., Engin, T., Baş, A., Yeter, A. Numerical and Experimental Investigation of the Effect on Heat Transfer of Nanofluid Usage in Mini/Micro Channels. Hittite Journal of 
Science \& Engineering. 5 (2018) doi: 10.17350/HJSE19030000113.

8. Orbay, S., Ozcelik, A., Bachman, H., Huang, T.J.T.J. Acoustic actuation of in situ fabricated artificial cilia. Journal of Micromechanics and Microengineering. 28(2) (2018) 025012. doi: 10.1088/1361-6439/aaa0ae.

9. Ayan, B., Ozcelik, A., Bachman, H., Tang, S.-Y.S.-Y., Xie, Y., Wu, M., et al. Acoustofluidic coating of particles and cells. Lab Chip. 16(22) (2016) 4366-72. doi: 10.1039/C6LC00951D.

10. Ozcelik, A., Nama, N., Huang, P.-H.H., Kaynak, M., McReynolds, M.R.M.R.M.R., Hanna-Rose, W., et al. Acoustofluidic Rotational Manipulation of Cells and Organisms Using Oscillating Solid Structures. Small. 12(37) (2016) 5120-5. doi: 10.1002/ smll.201601760.

11. Ahmed, D., Ozcelik, A., Bojanala, N., Nama, N., Upadhyay, A., Chen, Y., et al. Rotational manipulation of single cells and organisms using acoustic waves. Nature Communications. 7 (2016) 1-11. doi: 10.1038/ncomms11085.

12. Gupta, B., Rezai, P. Microfluidic Approaches for Manipulating, Imaging, and Screening C. elegans. Micromachines. 7(7) (2016) 123. doi: $10.3390 / \mathrm{mi} 7070123$.

13. Ben-Yakar, A., Chronis, N., Lu, H. Microfluidics for the analysis of behavior, nerve regeneration, and neural cell biology in C. elegans. Current Opinion in Neurobiology. 19(5) (2009) 561-7. doi: 10.1016/j. conb.2009.10.010.

14. Cheng, C.M., LeDuc, P.R., Lin, Y.W. Localized bimodal response of neurite extensions and structural proteins in dorsal-root ganglion neurons with controlled polydimethylsiloxane substrate stiffness. Journal of Biomechanics. 44(5) (2011) 856-62. doi: 10.1016/j. jbiomech.2010.12.006.
15. Massie, M.R., Lapoczka, E.M., Boggs, K.D., Stine, K.E., White, G.E. Exposure to the metabolic inhibitor sodium azide induces stress protein expression and thermotolerance in the nematode Caenorhabditis elegans. Cell Stress \& Chaperones. 8(1) (2003) 1. doi: 10.1379/1466-1268(2003)8<1:ETTMIS>2.0.CO;2.

16. Yanik, M.F., Cinar, H., Cinar, H.N., Chisholm, A.D., Jin, Y., BenYakar, A. Neurosurgery: functional regeneration after laser axotomy. Nature. 432(7019) (2004) 822. doi: 10.1038/432822a.

17. Garrity, P.A., Goodman, M.B., Samuel, A.D., Sengupta, P. Running hot and cold: behavioral strategies, neural circuits, and the molecular machinery for thermotaxis in C. elegans and Drosophila. Genes \& Development. 24(21) (2010) 2365-82. doi: 10.1101/ gad.1953710.

18. Chuang, H.-S., Chen, H.-Y., Chen, C.-S., Chiu, W.-T. Immobilization of the nematode caenorhabditis elegans with addressable lightinduced heat knockdown (ALINK). Lab on a Chip. 13(15) (2013) 2980. doi: 10.1039/c3lc50454a.

19. Zhang, P., Bachman, H., Ozcelik, A., Huang, T.J. Acoustic Microfluidics. Annual Review of Analytical Chemistry. 13(1) (2020) 17-43. doi: 10.1146/annurev-anchem-090919-102205.

20. Nawaz, A.A., Chen, Y., Nama, N., Nissly, R.H.R.H., Ren, L., Ozcelik, A., et al. Acoustofluidic Fluorescence Activated Cell Sorter. Analytical Chemistry. 87(24) (2015) 12051-8. doi: 10.1021/ acs.analchem.5b02398.

21. Ozcelik, A., Ahmed, D., Xie, Y., Nama, N., Qu, Z., Nawaz, A.A.A.A., et al. An Acoustofluidic Micromixer via Bubble Inception and Cavitation from Microchannel Sidewalls. Analytical Chemistry. 86(10) (2014) 5083-8. doi: 10.1021/ac5007798. 